Relations industrielles

Industrial Relations

\title{
II - La nécessité des réformes de structure \\ La structure de l'entreprise
}

\section{Marcel Clément}

Volume 3, numéro 6, février 1948

URI : https://id.erudit.org/iderudit/1023597ar

DOI : https://doi.org/10.7202/1023597ar

Aller au sommaire du numéro

Éditeur(s)

Département des relations industrielles de l’Université Laval

ISSN

0034-379X (imprimé)

1703-8138 (numérique)

Découvrir la revue

Citer cet article

Clément, M. (1948). II - La nécessité des réformes de structure : la structure de l'entreprise. Relations industrielles / Industrial Relations, 3(6), 82-86.

https://doi.org/10.7202/1023597ar

Tous droits réservés @ C Département des relations industrielles de l’Université Laval, 1948
Ce document est protégé par la loi sur le droit d'auteur. L'utilisation des services d’Érudit (y compris la reproduction) est assujettie à sa politique d'utilisation que vous pouvez consulter en ligne.

https://apropos.erudit.org/fr/usagers/politique-dutilisation/ 


\section{Bulletin des relations industrielles}

Volume 3, numéro 6

Février 1948 publié par
le Département des relations industrielles
de Laval.

T.R.P. Georges-H. LEvesQue, o.p., doyen.

Gérard Tremblay, directeur.

Abbé Gérard Dion, secrétaire.

Charles BELANGER et Jean GaGNE, assistantssecrétaires.

\section{ABONNEMENT:}

Etranger \$2.00

Autorisé comme envoi postal de seconde classe, Ministère des Postes, Ottawa.

Adressez toute correspondance au Secrétaire

Département des relations industrielles, Faculté des sciences sociales,

Université Laval,

\section{SOMMAIRE}

Notre troisieme congrès.. .. . . .. . . . ..

La structure de l'entreprise

Marcel Clément..

Nos collaborateurs....$\quad$..

The structure of enterprise

Marcel ClEment.. .. ..

La compensation des accidents du travail

Frédéric-T. Hecker..

Troisième congrès des relations indus-

trielles. - Programme..

Our third annual Convention.. .. .. .. 96

\section{II.-LA NECESSITE DES REFORMES DE STRUCTURE}

\section{LA STRUCTURE DE L'ENTREPRISE \\ Marcel CLEMENT}

Lorsque la construction d'une maison est achevée, nul n'en distingue plus les poutres ni les moellons. C'est pourtant la charpente, cachée par les revêtements extérieurs, qui donne à l'édifice sa forme et son assise. Cette charpente, l'architecte en a fait le plan. S'il fait une erreur, la construction en portera la trace. Si l'erreur est grave, il peut arriver que la maison s'écroule.

On constate un principe analogue dans les phénomènes vivants. Au fur et à mesure qu'un organisme se développe, les cellules initiales se multiplient et se différencient. Certaines d'entre elles deviennent des cellules osseuses. Elle se durcissent au fur et à mesure qu'elles deviennent $l e$ squelette, qui, bien que non apparent, donne à tout corps vivant sa silhouette et sa solidité. Pour chaque espèce vivante, le squelette a une forme parfaitement adaptée au fonctionnement des divers organes. Ainsi, il collabore à un plan d'ensemble qui conditionne la vie de l'organisme, mais de façon spontanée et évidemment inconsciente. La perfection même de cette adaptation résulte d'une finalité, ou pensée directrice, qui vient de l'Auteur de toute vie et non du hasard ou de la volonté de l'être vivant.
De même, toute société repose sur des structures. Comme la charpente disparaît dans les murs de la maison, et comme le squelette se devine à peine sous les muscles et l'épiderme, les structures sociales demeurent enveloppées dans les images et les agitations du monde extérieur. Elles n'en existent pas moins, et sont soumises à des lois propres. Nous voudrions tenter, dans cette étude, en premier lieu, de dégager les lois qui conditionnent toute structure sociale, puis de rechercher qu'elle est la structure économique de l'entreprise, enfin de dégager, à la lumière des résultats obtenus, le sens et la portée de ce que l'on a aujourd'hui pris l'habitude d'appeler: les réformes de structure.

\section{I}

La construction, la structure osseuse et la structure sociale ont en commun trois caractères, qui sont les caractères attachés à la notion même de structure :

a-la structure soutient, comme un cadre, la réalité structurée : la maison sans charpente, le corps sans squelette, la société sans institution s'effondrent; 
b-la structure maintient fixement la réalité structurée: au contraire des revêtements de la maison, des expressions du visage humain ou des modes passagères qui, mobiles, sont soumises au changement, la structure demeure;

c-la structure contient un plan préconçu, elle matérialise le schéma essentiel d'une idée directrice: plan de l'architecte, idée du Créateur de la vie, idéal politique, social et économique de l'homme.

De ces caractères communs, nous pouvons conclure que la structure sociale, comme toute structure, sera le soutien permanent et organisé, le cadre fixe et intentionnel, de la réalité sociale. Cette définition, toutefois, ne précise pas ce qui est particulier à la structure sociale.

Comparée à la structure biologique du squelette, la structure sociale, en effet, semble avoir une nature particulière. Le squelette réalise une idée directrice, mais cette idée directrice lui est imposeé par Dieu. D'où la perfection d'adaptation du squelette de chaque espèce animale aux conditions de vie de cet animal. Au contraire, la structure sociale, qui réalise aussi une idée directrice, reçoit cette idée directrice de l'homme. D'où les imperfections d'adaptation, plus ou moins graves, d'où les retouches permanentes, d'où les chambardements périodiques (révolutions) qu'ex-. priment et que subissent les structures sociales. Ainsi, ce qui différencie les structures sociales des constructions matérielles et des squelettes des corps vivants, c'est leur imperfection consécutive à la double condition de lhomme qui est libre, mais qui est déchu. Le caractère de permanence est donc moins accentué dans la structure sociale que dans les autres structures: il y a possibilité de réformes de structure.

Ainsi, notre définition se précise : le structure sociale devient le soutien permanent de la réalité sociale, tel que l'organise la liberté humaine.

Comment s'exerce la liberté humaine sur les structures sociales? Par l'invention d'une conception idéale de l'homme et l'adhésion à cet idéal. Ainsi les structures de la Rome antique ont été édifiées en accord avec l'idéal du Civis romanus, dans lequel chaque homme projetait ses tendances, ses passions, les meilleures et les pires. De même, la révolution de 1789 en France est précédée d'une intense fermentation littéraire, philosophique, intellectuelle. On élabore ainsi, en sollicitant de la liberté humaine l'adhésion des esprits et des volontés, un nouvel idéal de l'Homme et ce, au moment même où, de toute évidence, le structure de la France monarchique devait être adaptée à des conditions sociologico-économiques nouvelles. C'est en accord avec cet idéal que les structures institutionnelles qui sont nées de la Révolution ont été édifiées et codifiées. On sait comment Renan, Renan lui-même, a décrit cette «structure »: \&un code de loi qui semble avoir été fait pour un citoyen idéal, naissant enfant trouvé et mourant célibataire; un code qui rend tout viager, où les enfants sont un inconvénient pour le père, où toute oeuvre collective et perpétuelle est interdite, où les unités morales, qui sont les vraies, sont dissoutes à chaque décès, où l'homme avisé est l'égoïste qui s'arrange pour avoir le moins de devoirs possible, où la propriété est conçue, non comme une chose morale, mais comme l'équivalent d'une jouissance, un tel code, dis-je, ne peut engendrer que faiblesse et petitesse. 》

On a dans ce texte, indépendamment du jugement de valeur qu'il porte, une analyse complète du fonctionnement de la structure.

D'une part, cette structure \&semble avoir été faite pour un citoyen idéal ». Ainsi, c'est bien une conception idéale de l'homme dont la structure exprime l'idée directrice, dont elle fixe la règle et dont elle maintient l'application.

D'autre part, la structure dont parle Renan «ne peut engendrer que. . .» Ainsi, non seulement la structure reflète une pensée, mais elle engendre. Elle est un moule où les hommes vont être assouplis, formés, informés, déformés ou reformés, selon qu'ils s'y soumettront ou se rebelleront, mais un moule rigide, qui va soumettre la vie sociale de chacun et de tous.

Et, si la liberté humaine, par le choix et l'adhésion à un idéal, peut, lors des crises, qu'elle provoque, modifier les structures, ces structures en dehors des moments de crises, tendent à déterminer la conduite des hommes. Sans doute, chaque personne reste libre de ses choix et de ses actes. Toutefois, le nombre des choix pratiquement possibles se trouve limité et les lois statistiques expriment le déterminisme social, à l'échelle d'une collectivité, dans une structure donnée.

Au terme de cette brève analyse, nous pouvons donc définir la structure sociale comme : le cadre fixe d'une réalité sociale: a) organisée par la liberté humaine en fonction dune conception idéale de lhomme b) déterminant, par des impératifs positifs ou négatifs (de faire ou de ne pas faire) le nombre et le sens des choix personnels possibles dans la société ainsi construite. 


\section{II}

L'entreprise moderne est soutenue par une structure économique, qui reproduit, à son échelle, la structure nationale ou continentale du régime capitaliste néo-libéral dans lequel elle s'est développée. Quels sont donc, à l'échelle de l'entreprise, ${ }^{1}$ les idéaux du libéralisme, la structure suscitée par ces idéaux, quelles sont enfin les lois déterminées par cette structure?

Les économistes que l'on appelle encore « classiques », considéraient, idéalement, le travail comme une marchandise. Il n'y aurait pas là, d'ailleurs, une affirmation d'ordre philosophique. Simplement, un abus d'abstraction et de déduction logique. On est ensorcelé, au dix-huitième siècle, par la loi de l'offre et de la demande qui, récemment découverte, devient avec Adam Smith, la Loi et les Prophètes de l'économie politique. . On confond sa valeur positive avec un impératif normatif et l'on décrit avec complaisance pourquoi et comment le marché des denrées sera harmonieusement équilibré par cette loi. Mais le marché des denrées, c'est le lieu de rencontre des producteurs et des consommateurs, c'est là que se fixent les prix. Or, la logique de ce système exigeait une fixation aussi automatique et aussi perfectionnée des salaires. La tentation était forte, et nul n'y résista, de plaquer sur l'Entreprise, lieu de rencontre du Capital et du Travail, le schéma où l'on avait idéalement décrit le fonctionnement du marché, entre producteurs et consommateurs. «Le bien des travailleurs demande que la loi de l'offre et de la demande fonctionne sans entrave. Si les salaires baissent, c'est qu'il y a trop de demandes de travail dans le métier; abandonnés à eux-mêmes, les ouvriers se reporteront vers un métier mieux rétribué, et les salaires remonteront dans le premier, tandis qu'ils baisseront dans l'autre jusqu'à s'égaliser au point d'équilibre du salaire naturel ou normal. ${ }^{2}$ Il résulte effectivement de ce raisonnement que, pour le bien même du travailleur, le travail doit être traité comme une marchandise. Adam Smith est sur ce point d'une précision qui ne laisse aucun doute : «Deux quantités de travail, quel que soit le lieu, dit-il, sont d'égale valeur pour celui qui travaille. $\gg^{3} \mathrm{Du}$ point de vue économique donc, il suffirait de laisser le travail se conduire, ou mieux : être traité

(1) Rappelons que Yon distingue habituellement a) l'échelle de l'entreprise; b) l'échelle de la profession; c) l'échelle de la nation.

(2) Description de J. LECLERC: Leçons de Droit Naturel. IV, 225.

(3) A. SMrrH: Recherches sur la nature et sur les causes de la richesse, Ed. 1927, T. V, p. 125. comme une marchandise, il suffirait d'édifier, dans l'entreprise, un véritable «marché du travail», et la loi de l'offre et de la demande étendrait son influence régulatrice à tous les secteurs de la vie économique.

Pour correspondre à cet idéal, l'entreprise fut effectivement organisée en marché. Ni règlementation professionnelle, ni intervention de l'Etat ne sont tolérées, pendant la plus grande partie du dix-neuvième siècle, tant en France qu'en Angleterre. La structure fonctionne librement. Mais son fonctionnement ne correspond pas à l'image rêvée par Adam Smith. En effet, la concurrence met, au départ, l'employeur dans la nécessité de ne considérer les salaires des ouvriers que comme l'un des postes de son prix de revient. Son profit augmente lorsque le prix de revient diminue et que le prix de vente croît. Ainsi, salaires et profits varient nécessairement en fonction inverse l'une de l'autre. Dans une structure où la régulation est entièrement suspendue à la conquête du profit maximum, l'entrepreneur, ne rencontrant aucune entrave, est donc incité à écarter, par tous les moyens, les deux branches d'une tenaille dont les mâchoires écrasent, en sens inverse, le salariéconsommateur saisi entre les bas-salaires et les prix-élevés. ${ }^{4}$ Et ce que les libéraux avaient omis de prévoir, apparaît alors dans un jour cruel : à savoir que la marchandise qui s'achète d bas prix n'est pas pour autant modifiée, alors que le travail qui s'achète à bas prix, n'étant pas inerte, comme les denrées, mais inséparable de l'homme vivant qui le fournit, engendre de la souffrance et peut déchaîner une série de réactions directement contraires à l'harmonie espérée.

Ces réactions, qui se succèdent depuis la seconde moitié du dix-neuvième siècle, ont abouti à la constitution de syndicats, d'abord tolérés, puis acceptés et réglementés par la loi.

Quels sont les buts de ces syndicats? De garantir la sécurité du travailleur contre les effets désastreux du mécanisme inconscient de la loi de l'offre et de la demande. Ainsi il y a, de plus en plus, un «monde du travail» qui a son armée : le syndicat, son matériel de guerre : la grève, son traité de paix : la convention collective, son tribunal d'arbitrage : l'Etat. L'action syndicale, en outre, est bien placée pour suggérer des lois telle que la fixation d'un salaire minimum vital, ou toutes mesures générales d'intérêt pour le monde du travail.

Il va de soi que ces réalisations, correspondant aux aspirations de justice de la classe ou-

(4) Voir à ce propos, l'enquête ofticielle de Vuillermé. 
vrière, opèrent, au fur et à mesure qu'elles se développent, le début d'une véritable réforme de structure. Cette réforme aboutit à une situation économique que l'on a appelée «monopole bilatéral » et à propos duquel $\mathbf{H}$. Denis écrit: * En régime de monopole bilatéral..., bien que des échanges avantageux pour les deux parties puissent être réalisés, il ne peut pas y avoir d'échanges effectifs parce qu'il n'en existe pas qui soient considérés à la fois par les deux parties comme plus avantageux que le conflit. $\gg^{5}$ Est-ce à dire que la grève sera inévitable ? Nullement. Toutefois, l'expérience montre qu'elle est relativement fréquente et que l'accord spontané entre les deux monopolistes (travail et capital) est relativement rare. En effet, la structure du monopole bilatéral sur le marché du travail telle qu'elle résulte de l'action syndicale détermine une volonté d'entente, et c'est l'immense acquisition positive de ce régime, mais cette entente est conçue comme le résultat d'une épreuve de force, soit par simple pression morale (exposé des motifs) soit par des actes (grève, lock-out). Ainsi, le salarié reste, psychologiquement, dans cette structure l'antagoniste de l'employeur. Il a les moyens d'obtenir la justice par la réglementation collective, mais le ressort de ce moyen est une opposition, et non une composition. Capital et travail demeurent l'un en face de l'autre, de part et d'autre de la ligne où leurs intérêts s'affrontent: en définitive, les salaires varient toujours en fonction inverse du prix de revient, les salariés ne sont, en aucune façon, associés, économiquement, intellectuellement et moralement, à l'effort de production. Or, le syndicalisme patronal et ouvrier ne pourra, à l'échelon national, réaliser l'unité, que lorsqu'à la base même du système: l'entreprise, employeurs et travailleurs cessant d'être, à travers les conventions même collectives, acheteur et vendeur de travail, seront devenus compagnons de labeur et d'espoir. La réforme de la structure libérale n'aura donc atteint le coeur du problème que lorsqu'elle aura converti et dépassé Popposition qui demeure au sein de la cellule élémentaire de la structure: l'entreprise.

\section{III}

L'idéal du libéralisme a engendré, dans l'entreprise, une structure qui, trop souvent, a donné à la réalité une silhouette de laideur et qui a fait de chacun de ses gestes une souffrance sociale. Existe-t-il ùn idéal susceptible de s'exprimer dans des structures qui soutiendrait une réalité mieux conforme à la nature personnelle et sociale de

(5) H. DENIs: Le monopole bilatéral, p. 88. lhomme? Nous le savons bien, nous qui avons la volonté d'instaurer un ordre social chrétien qui vise précisément à bâtir une Cité à la taille des hommes sauvés par et dans l'Homme-Dieu. Mais avons-nous suffisamment pris conscience de ce que la réforme de la structure de l'entreprise était une pièce fondamentale, la pièce fondamentale, la charpente même de l'édifice.

Qu'on ne s'y trompe pas, en effet. Le programme que tracent les Encycliques ne conduit pas seulement à 《limiter les dégâts 》 du libéralisme. Lorsque Pie XI, dans \&Quadragesimo Anno » évoque l'enseignement de Léon XIII, c'est pour rappeler \&qu'il renversait si audacieusement les idoles du libéralisme » qu'il «provoqua, même chez certains catholiques, de la défiance, voire du scandale ». ${ }^{6}$ Et comment Léon XIII renversait-il les Idoles du Libéralisme? Dans les termes suivants, si denses de vérité et de foi: «Dans le corps humain, les membres, malgré leur diversité, s’adaptent merveilleusement l'un à l'autre, de façon à former un tout exactement proportionné et qu'on pourrait appeler symétrique. Ainsi, dans la société, les deux classes sont destinées par nature d s'unir harmonieusement dans un parfait équilibre. Elles ont un impérieux besoin lune de lautre: il ne peut y avoir de capital sans travail, ni de travail sans capital. $\gg^{7}$

La notion du travail-marchandise telle qu'elle résulte de l'économie libérale est-elle compatible avec cette union harmonieuse dans un parfait équilibre? \& Bien que le travail, ainsi que l'exposait nettement Notre Prédécesseur dans son Encyclique, ne soit pas une simple marchandise, qu'il faille reconnaître en lui la dignité humaine de l'ouvrier et qu'on ne puisse pas l'échanger comme une denrée quelconque, de nos jours, sur le marché du travail, l'offre et la demande opposent les parties en deux classes, comme en deux camps; le débat qui s'ouvre transforme le marché en un champ clos où les deux armées se livrent un combat acharné. A ce grave désordre qui mène la société à la ruine, tout le monde le comprend, il est urgent de porter remède. ${ }^{8}$

L'organisation des syndicats, la négociation des conventions collectives et l'arbitrage de l'Etat ne constituent-ils pas ces remèdes ? Toute la lettre de la Sacrée Congrégation du Concile, du 5 juin 1929, à Mgr Lienart, est là pour l'affirmer et l'établir. Toutefois, cette étape est-elle définitive? Il ne semble pas, car \& on ne saurait arriver à une

(6) Toutes les références aux textes pontificaux sont extraits du livre de documents publié par Chevalier et Marny: no 478.

(7) No 404.

(8) No 519. 
guérison parfaite que si, à ces classes opposées, on substitue des organes bien constitués, des « ordres » ou des «professions » qui groupent les hommes non pas d'après la position qu'ils occupent sur le marché du travail, mais d'après les différentes branches de l'activité sociale auxquelles ils se rattachent. ${ }^{9}$ Les conventions collectives conclues par les syndicats atteignent-elles ce but? Pas entièrement. Elles sont un traité de paix entre deux puissances. Elles ne sont pas encore la fédération de ces deux puissances s'unissant «harmonieusement dans un parfait équilibre». En effet, il y a toujours la tentation, pour chacune des puissances ouvrières et patronales ainsi organisées à l'échelle de la nation de régler les problèmes économiques à cette même échelle: ce fut l'erreur de la «carta di laboro » italienne, l'erreur de la loi du 4 octobre 1941 en France, l'erreur de certaines nationalisations plus récentes. ${ }^{10}$ Il y a, en autre, le danger de la création d'une psychologie collective de revendication et de contre-revendication. Les « organes bien constitués » groupant «les hommes non pas d'après la position qu'ils occupent sur le marché du travail, mais d'après les différentes branches de l'activité sociale auxquelles ils se rattachent » ne pourront donc pas être édifiés du premier coup à l'échelon de la profession ou de la nation. Comment réussirait-on à susciter, dans le corps tout entier, une structure qui ne serait pas au préalable réalisée dans chaque cellule ? Et n'est-il pas bien évident, au contraire, que si l'harmonie entre le Travail et le Capital était favorisée au sein de toutes les entreprises, par la structure même de l'entreprise, ces « organes bien constitués » en résulteraient tout naturellement dans chaque secteur professionnel, et dans la nation tout entière ? Et n'est-ce pas cette réforme de structure de l'entreprise qu'envisageait Pie XI lorsqu'il conseillait de «tempérer quelque peu, dans la mesure du possible, le contrat de travail par des éléments empruntés au contrat de société. »? Au moment où la contagion du dirigisme fait trembler les défenseurs de la «libre entreprise », n'est-ce pas là, en outre, un moyen efficace de sauver l'initiative personnelle, si légitime, des empiètements étatiques en y asso-

(9) No 519 .

(10) Voir à ce propos la lettre de S.S. Pie XII du 10 juillet 1946 à M. Charles Flory, président des Semaines sociales de France. ciant étroitement tous ceux qui coopèrent à la vie de l'entreprise. La plupart des pays d'Europe ont vu le dirigisme étatique d'inspiration socialiste succéder à la concurrence individuelle d'inspiration libérale. Cette seconde expérience, fille de la première, n'a pas mieux réussi qu'elle et c'est de ce second échec que sont nées toutes les expériences en cours, en vue de réformer la structure de l'entreprise. Un tel exemple est évidemment un thème de méditation.

Tel est donc l'idéal que nous propose la doctrine sociale chrétienne. Il n'est pas besoin d'insister sur le fait, qui sera ultérieurement développé, qu'en « tempérant le contrat de travail par des éléments empruntés au contrat de société », on dépasse, et on limite au sein de l'entreprise, l'antagonisme économique du profit et des salaires. Désor. mais, la « part du travail », et la « part du capital » varieront dans le même sens. ${ }^{11}$ Le travail n'est donc plus traité comme une marchandise: sa rémunération économique n'est plus seulement un contrat, individuel ou collectif, entre un vendeur et un acheteur. Patron et ouvriers sont, en quelque manière, associés. La «structure » répond ainsi à l'idéal de réconciliation, de composition et d'unité sociale. Nul doute que les fruits soient bons. N’appartient-il pas à la province de Québec de donner, sur le continent américain, le témoignage public de la valeur efficace de la doctrine sociale de l'Eglise?

On dira: «Mais qui ?»

Le Pape répond: «Les premiers apôtres, les apôtres immédiats des ouvriers seront des ouvriers, les apôtres du monde industriel et commerçant seront des industriels et des commerçants $\gg .{ }^{12}$

(11) “Dans le groupe normal ou la société normale, écrit à ce propos Joseph ScHuMPETER, ces éléments antagonistes s'intègrent avec les éléments coopératifs d'une manière harmonieuse dans le cadre d'une culture et d'une foi commune". Le contrat de société a précisément pour but de réaliser cette "intégration" des éléments coopératifs.

(12) No 556 .

\section{NOS COLLABORATEURS}

Clément, Marcel, licencié ès lettres, diplômé d'études supérieures de philosophie (Sorbonne), licencié en droit. Diplômé d'études supérieures d'économie politiques (Faculté de droit de Paris), professeur à la Faculté des sciences sociales de Laval.

HECKER, Frédéric-T., B.A., LL.L., avocat, Secrétaire de la Commission des accidents du travail, professeur à la Faculté des sciences sociales de Laval.

\section{CONVENTION COLLECTIVE - SÉCURITÉ SYNDICALE= \\ Le Rapport du deuxième Congrès des relations industrielles de Laval (1947), contenant le texte des conférences prononcées en cette occasion, est maintenant en vente au prix de $\$ 1.25$ (franco) au Département des relations industrielles de la Faculté des sciences sociales, 2, rue de l'Université, Québec.}

